\title{
Atividade antimetastática e antineoplásica do monterpeno 1,8-cineol: um teste in silico
}

\section{Anti-metastatic activity and the antineoplastic monterpene 1.8-cineole: a test in silico}

\author{
Ingrid Carneiro Cavalcante Souto \\ Graduanda de Odontologia da Universidade Federal de Campina Grande (UFCG) - Campus Patos/PB \\ E-mail: ingrid_ccsouto@hotmail.com \\ Emanuelle Ferreira Alves \\ Graduanda de Odontologia da Universidade Federal de Campina Grande (UFCG) - Campus Patos/PB \\ E-mail: emanuelle-alves13@hotmail.com \\ José Lucas Soares Ferreira \\ Graduando de Odontologia da Universidade Federal de Campina Grande (UFCG) - Campus Patos/PB \\ E-mail: jlucas_sf@hotmail.com
}

Heloísa Mara Batista Fernandes de Oliveira

Farmacêutica-Bioquímica do Hospital Universitário Ana Bezerra, Universidade Federal do rio Grande do Norte (UFRN)- Santa Cruz-RN. E-mail: heloisambf@gmail.com

Maria Angélica Satyro Gomes Alves

Professora Adjunta do Curso de Odontologia da Universidade Federal de Campina Grande (UFCG) - Campus Patos/PB. E-mail: angelicasatyro@hotmail.com

Abrahão Alves de Oliveira Filho

Professor Adjunto do Curso de Odontologia da Universidade Federal de Campina Grande (UFCG) - Campus Patos/PB. E-mail: abrahao.farm@gmail.com

Resumo: O câncer é caracterizado pelo crescimento descontrolado de células transformadas, que mesmo quando cessado o estímulo elas continuam a crescer, por isso é uma das maiores causas de mortalidade na atualidade. Diante dos altos índices de mortalidade se observa a necessidade cada vez mais intensa de tratamentos para o combate ao câncer. Além dos tratamentos já utilizados vêm sendo implementados tratamentos fitoterápicos a partir de óleos essenciais de plantas medicinais. Esses óleos essenciais são formados por misturas complexas de substâncias voláteis, que na composição desses óleos está um grupo de compostos chamados de terpenóides que tem um alto índice terapêutico. Dentre tantos terpenóides estudados objetivou-se realizar um estudo sobre a análise in silico da eficácia do monoterpeno 1,8-cineol na atividade metastática de tumores malignos. Os estudos in silico revelaram o potencial inibitório na metástase do monoterpeno 1,8-cineol, se apresentando como um importante antineoplásico e antimetastático.

Palavras-chave: Terpenoides. Monoterpeno. 1,8-cineol. Metástase tumoral.

\begin{abstract}
The cancer is characterized by the uncontrolled growth of cells transformed, that even when stopped the stimulus they continue to grow, so it is one of the leading causes of death in actuality. In the face of high rates of mortality observed the need increasingly more intense treatments for combating cancer. In addition to the treatments already in use have been implemented herbal treatments from essential oils of medicinal plants. These essential oils are formed by complex mixtures of volatile substances, which in the composition of these oils are a group of compounds called terpenoids which has a high therapeutic index. Among the many terpenoids studied aimed to carry out a study on the analysis in silico of the efficiency of monoterpene 1.8-cineole in metastatic activity of malignant tumors. The studies in silico revealed the inhibitory potential on the metastasis of monoterpene 1.8-cineole, introducing yourself as an important antineoplastic and anti-metastatic.
\end{abstract}

Key words: Terpenes. Monoterpene. 1,8-cineole. Neoplasm metastasis. 


\section{INTRODUÇÃO}

Doenças crônicas não transmissíveis como o câncer, estão se tornando cada vez mais importantes causas de morte e incapacidade com o decorrer do anos nos países desenvolvidos e em desenvolvimento (ALBINI et al., 2016). O câncer se caracteriza pela perda do controle da divisão celular e pela capacidade de invadir estruturas orgânicas, devido a anormalidade celular (INCA, 2011).

A manutenção e o crescimento dessas células formam os tumores malignos, que podem migrar do local de origem e se disseminar por todo o corpo, caracterizando uma metástase, podendo esta ser resistente ao tratamento e causar a morte do indivíduo (ALMEIDA et al., 2005).

Segundo o INCA (2014), estimativas indicam que a carga do câncer continuará crescendo nos países em desenvolvimento e crescerá ainda mais em países desenvolvidos se medidas preventivas não forem aplicadas. Em 2030, a carga global será de 21,4 milhões de casos novos de câncer e 13,2 milhões de mortes por câncer, em consequência do crescimento e do envelhecimento da população.

Diante desses altos índices de mortalidade e de previsões desfavoráveis, se observa a necessidade cada vez mais intensa de tratamentos para o combate ao câncer. Vale salientar a importância da observação do potencial de malignidade das células e o estágio que a doença se encontra, para assim se recomendar as medidas corretas de intervenção.

Além dos tratamentos com cirurgia, radioterapia e quimioterapia, vêm sendo implementados tratamentos fitoterápicos a partir de óleos essenciais de plantas medicinais que ajudam no combate a muitas doenças, inclusive o câncer. Dentre esses óleos essenciais, formados por misturas complexas de substâncias voláteis, está um grupo de compostos chamados de terpenoides o qual tem um alto índice terapêutico, o que dá a esses óleos essenciais a característica de fitoterápicos (SPITZER, 2004).

Os terpenos são compostos químicos oriundos do metabolismo secundário das espécies vegetais, constituindo uma rica fonte de compostos biologicamente ativos presentes em algumas plantas medicinais, como por exemplo, as plantas do gênero Eucalyptus. Os compostos terpênicos são divididos de acordo com o número de carbono em suas moléculas, em isoprenos ou hemiterpenos (5C), monoterpenos (10C), sesquiterpenos (15C); diterpenos (20C); sesterpenos (25C); triterpenos (30C); tetraterpenos (40C) e polisoprenóides (nC). Cada tipo de terpeno é estudado isoladamente e observado sua atividade farmacológica no metabolismo humano (CASTRO, 2010; SAAD et al., 2010; TEMPONE et al., 2008; SPITZER, 2004; BAKKALI et al., 2008).

Dentre tantos terpenóides destaca-se o monoterpeno 1,8 cineol, que é o principal composto do óleo do eucalipto, e que tem uma intensa atividade antiinflamatória, analgésica, antifúngica, anestésico e em especial, antineoplasica (AMARAL et al., 2004).

Objetivou-se analisar in silico a eficácia do monoterpeno 1,8-cineol na atividade metastática de tumores malignos na justificativa desta área possuir poucos estudos, além da sua beneficência e inovação.

\section{MATERIAL E MÉTODOS}

Para a realização dos estudos in silico, todas as informações químicas (estrutura química da molécula, massa molecular, polaridade, CAS-number) do monoterpeno selecionado (carvona) foram obtidas no site http://www.chemspider.com/.

A Previsão do espectro de atividade para substâncias (PASS) online é um software projetado para avaliar o potencial biológico geral de uma molécula orgânica in silico sobre o organismo humano, onde fornece previsões simultâneas de muitos tipos de atividades biológicas com base na estrutura dos compostos orgânicos. O espectro de atividade biológica de um composto químico é o conjunto de diferentes tipos de atividade biológica, que refletem os resultados de interação do composto com várias entidades biológicas. Pass online dá várias facetas da ação biológica de um composto, obtendo os índices Pa (probabilidade " de ser ativo") e Pi (probabilidade "de ser inativo") estimando a categorização de um composto potencial em ser pertencente à subclasse de compostos ativos ou inativos, respectivamente (SRINIVAS et al., 2014)

\section{RESULTADOS E DISCUSSÃO}

Os monoterpenos são um tipo de terpenóide composto de 10 unidades de carbono, apesar de possuírem uma estrutura simples eles estão presentes nos óleos essenciais de muitas plantas aromáticas, conferindo a essas plantas grande importância farmacológica (BHALLA et al., 2013; GUIMARÃES et al., 2013; SANTANA et al., 2013; RIELLA et al., 2012).

O 1,8-cineol que tem um importante efeito analgésico e anti-inflamatório, como também antimetastático, como mostra a Tabela 1.

Tabela 1 - Valores de Pa, Pi e atividade metastática para o monoterpeno 1,8-cineol.

\begin{tabular}{ccc}
\hline $\begin{array}{c}\text { Probabilidade de ser ativo } \\
(\mathbf{P a})\end{array}$ & $\begin{array}{c}\text { Probabilidade de ser inativo } \\
(\mathbf{P i})\end{array}$ & $\begin{array}{c}\text { Atividade terapêutica } \\
\text { (antineoplásica) }\end{array}$ \\
\hline 0,150 & 0,137 & Câncer de Mama \\
0,173 & 0,051 & Câncer de Colo de Útero \\
0,716 & 0,005 & Câncer de Cólon \\
0,721 & 0,005 & Câncer Colorretal \\
0,757 & 0,005 & Câncer de Pulmão \\
0,341 & 0,169 & Linfoma Não-hodking \\
0,605 & 0,005 & Atividade Antimetastática \\
\hline
\end{tabular}


Estudos in silico revelaram o potencial inibitório na metástase do monoterpeno 1,8-cineol, se apresentando como um importante antineoplásico em que na maioria dos testes apresentou um potencial de ativação $(\mathrm{Pa})$ do efeito antineoplásico bem mais elevado que o potencial de inativação (Pi). Especificamente como antimetastático ele apresenta um PA de 0,605 em relação a um Pi de 0,005.

Estes dados confirmam os resultados obtidos no estudo in vitro revelado por Badrhadad e colaboradores (2012). Partindo do pressuposto que a angiogênese é um acontecimento essencial no crescimento de tumores e metástases, se observou in vitro o efeito do Angustifolia elaeagnus e Nepeta crispa (espécies de plantas importantes que crescem no Irã) sobre as atividades anti-angiogênicas em células endoteliais umbilicais humanas (HUVEC). Especificamente a Nepeta crispa, que tem em sua composição 47.9\% de 1,8-cineol, em uma concentração de $400 \mathrm{ml}$ ela apresentou forte inibição de fatores de crescimento próantigênicos (BADRHADAD et al., 2012).

\section{CONCLUSÃO}

O monoterpeno 1,8-cineol tem grande potencial e eficácia na atividade antimetastática, tanto no estudo in silico como na observação in vitro os resultados foram bem positivos e favoráveis. No entanto, ainda existem poucos estudos nessa área, o que revela a necessidade de se intensificar mais estudos sobre o assunto.

\section{REFERÊNCIAS}

ALBINI, A.; DECENSI, A.; CAVALLI, F.; COSTA, A. Cancer prevention and interception: a new era for chemopreventive approaches. Clinical Cancer Research, v.22, n.14, p.1-15, 2016.

ALMEIDA, V. L.; ANDREI, L.; REINA, L. C. B.; MONTANARI, C. A.; DONNICI, C. L.; LOPES, M. T. P. Câncer e Agentes Antineoplásicos Ciclo-Celular Específicos e Ciclo-Celular não Específicos que Interagem com o DNA: Uma Introdução. Quim. Nova, v. 28, n. 1, p. 118-29, 2005.

AMARAL, J. F., SANTOS, F. A., SOUSA, F. C. F., ALMEIDA, F. Atividade antiinflamatória, antinociceptiva, e gastroprotetora do óleo essencial de Croton sonderianusMuell. Arg. Fortaleza: UFC, 2004, 150p.

BADRHADAD A.; PIRI KH; MANSOURI K. In vitro antiangiogenic activity fractions from hydroalcoholic extract of Elaeagnus angustifolia L. flower and Nepeta crispa L. arial part. Journal of Medicinal Plants Research, v.6, n.31, p. 46334639, 2012.

BAKKALI, F.; AVERBECK, S.; AVERBECK, D.; IDAOMAR, M. Biological effects of essential oils - A review. Food and Chemical Toxicology, v. 46, n.2, p.446475, 2008.

BHALLA, Y.; GUPTA, V. K.; JAITAK, V. Anticancer activity of essential oils: a review. Journal of the Science of Food and Agriculture, v. 93, n.15, p.3643-3653, 2013.
CASTRO, R. D. Atividade antifúngica do óleo essencial de CinnamomumzeylanicumBlume (Canela) e de sua associação com antifúngicos sintéticos sobre espécies de Candida. João Pessoa: UFPB, 2010, 169p.

GUIMARÃES, A. G.; QUINTANS, J. S. S.; QUINTANSJÚNIOR, L. J. Monoterpenes with Analgesic Activity-A Systematic Review. Phytotherapy Research, v. 27, n.1, p. 115, 2013.

INCA, INSTITUTO NACIONAL DE CÂNCER (BRASIL). ABC do câncer: abordagens básicas para o controle do câncer / Instituto Nacional de Câncer. Rio de Janeiro: Inca, 2011. 128 p. Disponível em: <http://bvsms.saude.gov.br/bvs/controle_cancer>. Acessado em: 20. Jul. 2016.

INCA, INSTITUTO NACIONAL DO CÂNCER. Estimativa 2014. Incidência de Câncer no Brasil. Disponível em: <http://www.inca.gov.br/estimativa/2014/index.asp?ID=2>. Acesso em: 25. Maio. 2016.

RIELLA, K. R.; MARINHO, R. R.; SANTOS, J. S.; PEREIRA-FILHO, R. N.; CARDOSO, J. C.; ALBUQUERQUE-JUNIOR, R. L. C.; THOMAZZI, S. M. Anti-inflammatory and cicatrizing activities of thymol, a monoterpene of the essential oil from Lippia gracilis, in rodents. Journal of Ethnopharmacology, v. 143, n.2, p.656$663,2012$.

SAAD, A.; FADLI, M.; BOUAZIZ, M.; BENHARREF, A.; MEZRIOUI, N.-E.; HASSANI, L. Anticandidal activity of the essential oils of Thymus maroccanus and Thymus broussonetii and their synergism with amphotericin B and fluconazol. Phytomedicine, v. 17, n. 13, p. 1057-1060, 2010.

SANTANA, M. T.; DE OLIVEIRA, M. G.; SANTANA, M. F.; DE SOUSA, D. P.; SANTANA, D. G.; CAMARGO, E. A.; DE OLIVEIRA, A. P.; ALMEIDA, J. R.; QUINTANS, L. J. Citronellal, a monoterpene present in Java citronella oil, attenuates mechanical nociception response in mice. Pharmaceutical Biology, v. 51, n. 9, p. 1144-1149, 2013.

SPITZER, C. M. O. S. V. Óleos voláteis. In: SIMÕES, C. M. O.; SCHENKEL, E. P.; GOSMANN, G.; MELLO, J.C.P.; MENTZ, L. A.; PETROVICK, P. R. Farmacognosia - da planta ao medicamento. $5^{\mathrm{a}}$ ed. Porto Alegre/Florianópolis: Editora da UFRGS/Editora da UFSC, p. 467-495, 2004.

SRINIVAS, N.; SANDEEP, K. S.; ANUSHA, Y.; DEVENDRA, B. N. In Vitro Cytotoxic Evaluation and Detoxification of Monocrotaline (Mct) Alkaloid: An In Silico Approach. Int. Inv. J. Biochem. Bioinform., v.2, n.3, p.2029, 2014.

TEMPONE, A. G.; SARTORELLI, P.; TEIXEIRA, D.; PRADO, F. O.; CALIXTO, I. A.; LORENZI, H.; MELHEM, M. S. Brazilian flora extracts as source of novel antileishmanial and antifungal compounds. Memórias do Instituto Oswaldo Cruz, v. 103, n. 5, p. 443-449, 2008. 